\title{
Reflexividade, foco e a vogal -i- do georgiano*
}

\author{
Reflexivity, focus and the Georgian vowel - $i$ -
}

João Paulo Lazzarini-Cyrino'

Aluno de Doutorado pela Universidade de São Paulo.

ipcyrino@gmail.com
RESUMO: 0 presente trabalho analisa as estratégias de reflexivização do georgiano sob a luz da proposta de Hornstein (1999, 2001) de satisfação de papéis temáticos por movimento, explicando - assim - o sincretismo do morfema -i- presente nessas construções e em verbos inacusativos (anticausativos/médios e passivos da língua). Essa análise vai contra o senso comum de que o morfema -i- é um operador de valência verbal ou voz (cf. Nash, 2002), mostrando que, na verdade, trata-se da realização de uma cópia incorporada do argumento interno do verbo. Palavras-chave: Reflexividade; Voz; Foco; Minimalismo.

ABSTRACT: The present paper analyses the reflexivization strategies in Georgian under Hornstein's $(1999,2001)$ theta-role checking by movement proposal as an attempt to explain the syncretism of the morpheme -i-, which occurs in reflexives, anticausatives and passives. This analysis goes against the idea that this morpheme is a valence or voice operator (cf. Nash, 2002), showing that it is rather the realization of an incorporated copy of the internal argument.

KEYworDS: Reflexivity; Voice; Focus; Minimalism.

* Projeto financiado pela FAPESP, processo no. 2011/21973-1. Este trabalho foi escrito no primeiro semestre de 2012 como condição de aprovação no curso de pósgraduação Introdução ao Minimalismo, ministrado pelo Prof. Dr. Jairo Nunes, USP. Agradeço aos pareceristas por uma interessantíssima discussão a respeito do sistema utilizado neste trabalho para representar foco nos constituintes. Não foi possível implementar as ideias sugeridas por questões de tempo e espaço. Atento aos leitores, portanto, que compreendam o sistema apresentado aqui como um caminho de análise e não como uma proposta definitiva. 


\section{Introdução}

roponho, neste artigo, um caminho possível para a compreensão do status do morfema pré-radical verbal -i- do georgiano. Trata-se de uma marca análoga ao $S E$ românico, ocorrendo em diversos contextos: anticausativos, médios, passivos e reflexivos. O caráter afixal dessa marca, no entanto, faz com que ela seja compreendida - à primeira vista - como um expoente de uma categoria funcional detransitivizadora (uma versão de $v$ ou Voice), conforme apontado por Nash (2002). No entanto, conforme mostro aqui, alguns fatos sugerem que -i-, na verdade, ocorre com verbos claramente transitivos, não podendo, assim, ser analisado como um detransitivizador.

A solução encontrada aqui é, seguindo algumas propostas dentro do Programa Minimalista (HORNSTEIN, 1999, 2001) e da Morfologia Distribuída (ALBOIU; BARRIE; FRIGENI, 2004), considerar que -i-é o expoente fonológico de uma inferior do sujeito dos verbos em que ocorre. Esse tipo de cópia é afixada ao verbo por meio da operação Morphological Merger (inicialmente proposta em Marantz, 1988). Desenvolve-se aqui que o motivador dessa afixação é a ausência de foco que essas marcas apresentam.

$\mathrm{O}$ artigo divide-se em duas partes: primeiramente mostro os dados e os problemas em se analisar -i- como um detransitivizador e posteriormente desenvolvo a análise introduzida acima.

\section{Sincretismo de -i- e a Análise Inacusativa dos Reflexivos}

Construções anticausativas/médias/passivas e reflexivas em georgiano têm seus verbos marcados com a vogal -i-. Porém, no caso dos verbos reflexivos, há também a ocorrência do nome reflexivo tav, que, literalmente, significa cabeça. Abaixo seguem exemplos de verbos anticausativos/médios/ $\operatorname{passivos}^{1}(1)$ e reflexivos (2) ${ }^{2}$.

(1) a. K'ar -i da-i-gh-a

Porta-ABS PVB-VPR-abrir-3sSUJ.

"A porta abriu."

b. Saxl-i i-tsveb-a.

Casa-nom VPR-queimar-3sSUJ

"A casa está queimando."

(2) a. K'ac-i tav-s i-k'ritik'eb-s.

Homem-NOM REFL-ACU VPR-criticar-3sSUJ.

"O homem se critica."

b. Shen tav-i i-zhag-e

Você.ERG REFL-ABS VPR-amaldiçoar-AOR

"Você se amaldiçoou."

Em Nash (2002), o morfema -i- é considerado como um detransitivizador, comparável ao SE românico em análises como em Bouchard (1984), Grimshaw (1990) e Marantz (1984)³. Há, à primeira vista, certa lógica para que -i- figure como um operador de valência verbal. Além de estar

As leituras anticausativas médias e passivas são obtidas com formas idênticas do verbo e nas sentenças exemplificadas em (1).

2 Abreviações nas glosas utilizadas para os dados do georgiano neste trabalho:

NOM Nominativo GEN Genitivo

DAT Dativo ACU Acusativo

ABS Absolutivo ERG Ergativo

INS Instrumental

TH Tema Verbal

AOR Aoristo

VPR Vogal Pré-Radical

REFL Nome Reflexivo

PVB Preverbo (tipo de prefixo verbal)

POSS Pronome Possessivo

2pSUJ Concordância do verbo com o Sujeito/Objeto.

3 O que há em comum entre essas análises do SE românico é considerar o clítico como um operado que reduz a valência verbal de alguma forma. Nesse tipo de análise os verbos reflexivos são, em última consequência, considerados intransitivos. 
relacionado à detransitivização de alguns verbos, o morfema é um expoente de um paradigma que a gramática tradicional nomeia vogais pré-radicais. As vogais pré-radicais encontram-se justamente indicando propriedades argumentais dos verbos, tais como a introdução de um argumento locativo ou beneficiário, como visto em (3) abaixo:

(3) a. Mama a-ts'er-s ts'eril-s kaghald-s.

Pai.NOM VPR-escrever-3sSUJ carta-ACU papel-DAT ${ }^{4}$.

"O Pai escreve a carta sobre o papel."

b. Is ga-u-k'et-a tavis deda-s.

Isso.ABS PVB-VPR-fazer-3sSUJ seu.DAT mãe-DAT.

"Ele fez isso para/pela sua mãe."

No entanto, se -i- é considerado um operador detransitivizador, para analisá-lo de maneira uniforme em seus contextos de ocorrência é necessária uma explicação para a transitividade das construções reflexivas em (2) com o objeto tav. Essa explicação aparentemente se encontra em Embick (2004), que lida com um fenômeno parecido em algumas reflexivas do grego: a ocorrência simultânea de um clítico reflexivizador afto com a concordância não-ativa (NAt), que é sincrética em reflexivos, anticausativos e passivos. Abaixo ilustra-se o fenômeno:

(4) O Iannis afto-katastraf-ike.

O João REFL-destruir-3s.NAt.

"O João se autodestruiu."

(EMBICK, 2004)

\footnotetext{
${ }^{4}$ Em georgiano as marcas de Dativo e de Acusativo são sincréticas. Detecta-se que um argumento é marcado por Acusativo ao invés de Dativo quando se alterna o sistema de casos para Ergativo/ Absolutivo.
}

Para Embick, esses casos são explicados por sua versão de Análise Inacusativa dos Reflexivos. O autor leva em conta a existência de um nó $v /$ Voice com as propriedades apontadas por Kratzer (1996), representadas da seguinte forma. Podem ocorrer dois traços em $v$, [Caso] e [Ag]. O primeiro permite que o verbo cheque caso Acusativo e, por sua vez, licencia um argumento externo, em conformidade com a Generalização de Burzio. 0 segundo permite a interpretação de um agente do evento verbal. Há então uma tipologia de $v$-s de acordo com a ocorrência desses traços:

(5) a. Transitivos: [Caso], [Ag]

b. Passivos: $[\mathrm{Ag}]$

c. Anticausativos: $\varnothing$

A questão, entretanto, é a respeito dos reflexivos. Qual é o seu lugar nessa tipologia? A resposta está no fato de que em algumas línguas, como o grego, há - de um modo geral - total identidade de formas entre sentenças passivas/anticausativas e reflexivas. Essas construções são intransitivas na língua e seu verbo é marcado, então, pela concordância não-ativa, que ocorre tanto em reflexivos como em anticausativos:

(6) a. To ktirio gkremiz-ete.

O prédio derrubar-3s.NAt.

"O prédio está caindo."

b. O Iannis xteniz-ete.

O João pentear-3s.NAt.

“O João está se penteando."

É possível observar essa semelhança de formas mesmo em português brasileiro, onde se formam tanto verbos reflexivos como alguns anticausativos apenas com a colocação do clítico SE junto ao verbo, como abaixo. 
(7) a. O menino se machucou.

b. O menino se elogiou.

Assim, sintaticamente, reflexivos e anticausativos são idênticos: não apresentam o traço [Caso] em $v$, não projetando argumentos externos. 0 clítico SE do português brasileiro, por exemplo, seria um expoente fonológico especificado para um $v$ sem [Caso] ${ }^{5}$. No entanto, deve-se explicar a ocorrência da concordância não-ativa do grego em dados como o em (4) repetido abaixo:

(4) O Iannis afto-katastraf-ike.

O João REFL-destruir-3s.NAt.

"O João se autodestruiu."

(EMBICK, 2004)

Para Embick, esse dado é inesperado levando em conta a generalização acima, uma vez que mostra uma construção transitiva - considerando-se afto como objeto do verbo - com a concordância não-ativa, que seria reservada apenas para verbos sem argumento externo. O autor explica esse dado primeiro considerando que essa é uma construção reflexiva sintaticamente gerada. As outras - intransitivas - são reflexivas inerentes, ou seja, a raiz verbal permite a interpretação reflexiva quando se encontra detransitivada. Em grego a Sintaxe pode gerar construções reflexivas com o anafórico clítico afto, que marcaria o agente da reflexiva, sendo gerado como argumento externo. O sujeito continua sendo gerado como argumento interno, que se alça à posição de sujeito para satisfazer os requisitos de ligação da anáfora afto - adjungida a $v$-, resultando uma derivação como a vista abaixo em (8):

\footnotetext{
${ }^{5}$ Essa análise se dá nos termos da Teoria da Morfologia Distribuída, em que se assume inserção tardia de fonologia. Essa inserção se dá por meio de regras de correspondência (Itens de Vocabulário) entre traços sintático-semânticos presentes nos nós terminais da derivação sintática e forma fonológica.
}

(8)

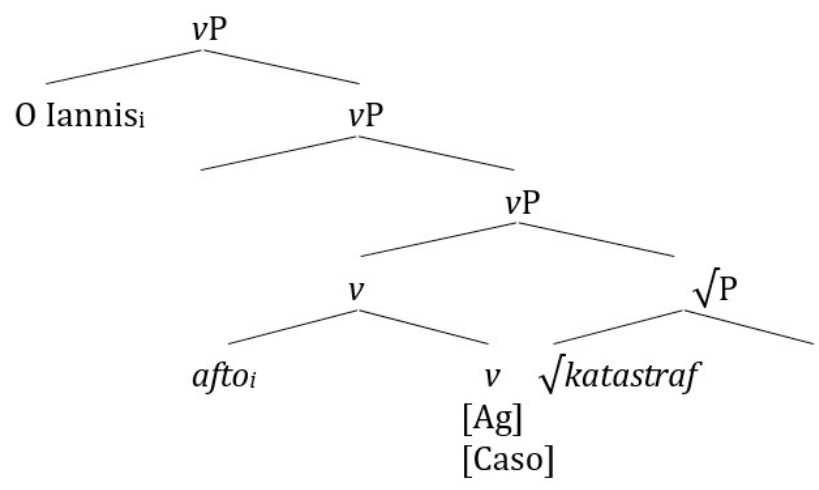

Nesse quadro, a concordância não-ativa continua sem ter sua ocorrência explicada em termos dos traços de $v$. Entretanto, há um recurso dentro da teoria da Morfologia Distribuída, adotado pelo autor, que consiste na inserção de traços posteriormente ao Spell-Out. Esses traços são, portanto, relevantes apenas para a construção da forma fonológica do conteúdo derivado pela sintaxe, não apresentando nenhuma interpretação. Embick diz que há em grego uma regra pós-sintática que insere um traço $[\mathrm{X}]$ em $v$ toda vez que não houver um argumento externo do verbo que seja um DP-pleno ${ }^{6}$. A concordância não-ativa seria então especificada para esse traço [X].

Se consideramos o tav do georgiano como um anafórico e anafóricos como DPs não-plenos, essa análise poderia explicar a inserção do morfema -i- em reflexivas e anticausativas. Uma regra pós-sintática inseriria um traço para o qual -i- estaria especificado no caso de não haver argumentos externos plenos do verbo: -i- seria inserido tanto nos anticausativos, que não apresentam argumento externo, como nos reflexivos, que apresentariam o argumento externo tav, não-pleno. No entanto, alguns problemas podem ser

6 Não é clara a definição de DP-pleno para o autor. No artigo, o autor cita como exemplo um DP não-clítico. Para a implementação dessa análise ao -i- georgiano considerarei também que um DP-pleno não pode ser anafórico. 
levantados contra essa análise. O primeiro deles é com relação ao status de tav enquanto anáfora.

Um fato que não foi levantado em Nash (2002) ao tratar -i- como um operador detransitivizador é o de que, em construções reflexivas, -i- pode alternar-se com um pronome possessivo. Sendo assim, há duas estratégias básicas de reflexivização de um verbo transitivo, ilustradas abaixo:

(9) a. Shen rat'om tav-s i-xedav?

Você.NOM por-que REFL-ACU VPR-OLHAR.

"Por que você está se olhando?"

b. Shen rat'om shen tav-s xedav?

Você.Nom por-que seu.ACu REFL-ACU olhar.

"Por que você está se olhando?"

Esses dados nos mostram que não é tav que se alterna com um elemento sem autonomia referencial (-R, nos termos de Reinhart \& Reuland, 1993), mas sim o morfema -i-. Ou seja, o nome reflexivo tav não pode ser considerado, sozinho, a anáfora da expressão, já que nesta há um elemento claramente -R.

Mas, mesmo supondo que tav seja um DP não-pleno, há construções transitivas em que -i- ocorre que não apresentam nenhum argumento externo não-pleno. São construções que apresentam duas leituras: a de que o sujeito possui o objeto e a de que o sujeito se beneficia com a ação sobre o objeto. Abaixo seguem exemplos:

$$
\begin{aligned}
\text { (10) a. } & \text { Dato i-ghebav-s saxl-s. } \\
& \text { Dato.Nom VPR-pintar-3sSUJ casa-ACU. } \\
& \text { "O Dato }{ }_{\mathrm{i}} \text { está pintando a sua }{ }_{\mathrm{i}} \text { casa." } \\
& \text { "O Dato }{ }_{\mathrm{i}} \text { está pintando a } \text { casa }_{\mathrm{i} / \mathrm{j}} \text { para }_{\mathrm{i}} \mathrm{i}_{\mathrm{i}} \text { " }
\end{aligned}
$$

b. Ana-m she-i-ch'r-a

tma.

Ana-ERG PVB-VPR-cortar-3sSUJ.AOR cabelo.ABS.

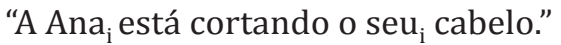

"A Ana $\mathrm{i}_{\mathrm{i}}$ está cortando o cabelo $\mathrm{i}_{\mathrm{i} / \mathrm{j}}$ para $\mathrm{si}_{\mathrm{i}}$ "

As mesmas duas leituras podem ser obtidas com um pronome possessivo ao invés de -i-, o que mostra que a leitura de sujeito beneficiário é inferida7

(11) a. Dato ghebav-s tavis saxl-s.

Dato.Nom pintar-3sSUJ seu.ACU casa-ACU.

"O Dato ${ }_{i}$ está pintando a sua $\mathrm{i}_{\mathrm{i}}$ casa."

"O Dato ${ }_{\mathrm{i}}$ está pintando a casa $\mathrm{i}_{\mathrm{i} / \mathrm{j}}$ para $\mathrm{si}_{\mathrm{i}}$ "

\section{b. Ana-m she-ch'r-a tavis-i tma}

Ana-ERG PVB-VPR-cortar-3sSUJ.AOR seu-ABS cabelo.ABS.

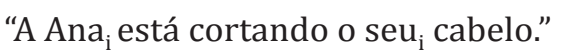

"A Ana ${ }_{\mathrm{i}}$ está cortando o cabelo $\mathrm{i}_{\mathrm{i} / \mathrm{j}}$ para $\mathrm{si}_{\mathrm{i}}$ "

A alternância possível de (10) para (11) é paralela à vista nas construções reflexivas em (9), o que mostra que (i) o morfema -i- assume o mesmo comportamento em ambas as construções e (ii) o morfema -i- está realmente relacionado a um papel anafórico e não ao de um operador de projeção argumental dos verbos.

Uma evidência contra o caráter de argumento externo de tav surge com o teste de caso absolutivo. Como o georgiano apresenta o sistema de casos ergativo/absolutivo no tempo aoristo, um teste para se detectar a natureza dos argumentos do verbo consiste em alternar a sentença para esse

Há outros argumentos para atestar que a leitura de beneficiário é inferida da de posse, porém, seria necessário observar outras construções da língua, o que não cabe neste trabalho. Para maiores esclarecimentos, ver Lazzarini-Cyrino (2011). 
tempo. Os argumentos que ficarem marcados com ergativo são argumentos externos, e os marcados com absolutivo são argumentos internos do verbo. Tavé realizado com caso absolutivo no aoristo, o que mostra que se trata, na verdade, de um argumento interno do verbo. Abaixo segue o teste:

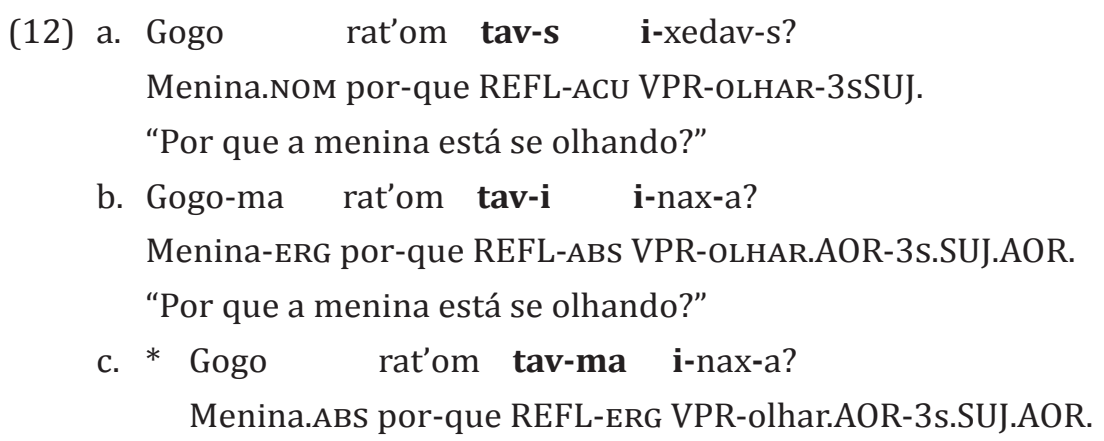

Um último problema, que se relaciona também à teoria de Embick de um modo geral, é o fato de se considerar que os reflexivos sem afto do grego devem ser tratados como reflexivos "inerentes". Em georgiano há também alguns verbos com leitura reflexiva que não apresentam tav. No entanto, esses verbos, além de não apresentarem tav, não apresentam também o morfema -i- e não se comportam como inacusativos. Curiosamente, esses verbos são raros na língua, ao contrário do que ocorre com o grego, onde as reflexivas com afto é que são raras. Abaixo segue um exemplo desses reflexivos "inerentes" do georgiano, com o teste de caso absolutivo sendo agramatical, evidenciando que seus sujeitos são argumentos externos, ou seja, tratam-se de verbos inergativos ${ }^{8}$ :

\footnotetext{
8 O comportamento dos verbos em (13) e (14) interessantemente coincide com o que Reinhart (2000) e Reinhart e Siloni (2005) descrevem acerca dos reflexivos inerentes serem verbos inergativos nas línguas e não produtivos. A análise proposta por Embick coloca o grego moderno como uma exceção a esse fenômeno trans-linguístico, algo que é ignorado pelo autor e requeria maiores esclarecimentos.
}

(13) a. Mariam-i banaob-s Maria-Nom lavar_se-3s.SUJ.

"A Maria está se lavando."

b. * Mariam-i i-bana-a.

Maria-ABS PVB-lavar_se-3s.SUJ.AOR.

c. Mariam-am i-ban-a.

Maria-ERG PVB-lavar_se-3s.SUJ.AOR.

"A Maria lavou-se."

Isso mostra, novamente, que não há paralelismo entre o comportamento de tav e o de afto que possa levar a considerá-los elementos correspondentes. O nome reflexivo tav é requerido para que haja reflexivização de qualquer predicado, ao contrário de afto, que ocorre improdutivamente em pouquíssimos verbos.

\section{Real Status do Morfema -i-}

Até agora reunimos os argumentos cruciais que apontam contra uma análise do morfema -i- como um detransitivizador. Dois fatos são problemáticos para a análise. $O$ primeiro é o de que tav não pode ser caracterizado como argumento externo do verbo, o que impede que a análise uniforme que a teoria de Embick ofereceria para o morfema -i- não possa ser aplicada. O segundo é a existência de construções transitivas com -i(dados em (10)), o que realmente não sugere uma análise de -i- como um detransitivizador. A pergunta, no entanto, é qual seria o real status da vogal pré-radical -i- do georgiano?

A resposta para essa pergunta parece ter relação com a alternância vista anteriormente entre -i- e pronomes possessivos: 
(14) a. K'ac-i tav-s i-k'ritik'eb-s.

Homem-Nom REFL-ACU VPR-criticar-3sSUJ.

"O homem se critica."

b. Shen tav-i i-zhag-e

Você.ERG REFL-ABS VPR-amaldiçoar-AOR

"Você se amaldiçoou."

(15) a. K'ac-i tavis tav-s k'ritik'eb-s.

Homem-NOM seu.ACU REFL-ACU criticar-3sSUJ.

"O homem se critica."

b. Shen shen-s tav-s zhag-e.

Você.ERG seu-ABS REFL-ABS amaldiçoar-AOR.

"Você se amaldiçoou."

Lubowicz (1999), ao estudar a distribuição de duas estratégias de reflexivização do polonês, os pronomes sie e siebie, mostra que a primeira forma não pode ser coordenada com outros DPs e não pode responder perguntas de foco sobre objeto ${ }^{9}$ :

(16) Kasia umyła siebie/*się i Ania.

Kasia lavou SE /*se e Ania.

"Kasia lavou a si própria e a Ania."

(Cf. Lubowicz, 1999, p. 11)

(17) Kogo myjesz? - Siebie/*się.

Quem lava? - SE/*se.

"Quem você lava? A mim próprio/*se."

(Cf. Lubowicz, 1999, p. 5)

9 Esses testes parecem se aplicar, na verdade, a várias línguas. Kapitonov (2004) mostra os mesmos testes para o Russo. Em um relatório apresentado ao $\mathrm{CNPq}$ mostro o mesmo para o português brasileiro, o grego moderno, o albanês e o próprio georgiano, que apresento aqui.
Para o georgiano (e para outras línguas, cf. nota 9) o mesmo comportamento é atestado: a forma -i- + tav não ocorre em coordenação com outros DPs, bem como não ocorre como resposta de pergunta focalizando o objeto:

(18) a. K'ac-i mariam-s da tavis tav-s k'ritik'eb-s. Homem-Nom Maria-ACU e seu.ACU REFL-ACU criticar-3sSUJ. "O homem critica a Maria e a si mesmo."
b. * K'ac-i
mariam-s da tav-s
i-k'ritik'eb-s.

Homem-Nom Maria-ACU e REFLX-ACU VPR-criticar-3sSUJ.

\section{(19) a. Vis k'ac-i k'ritik'eb-s? k'ritik'eb-s tavis tav-s.} Quem.ACU homem-NOM criticar-3sSUJ? criticar-3sSUJ seu.acu REFL-ACU. "Quem o homem critica? - critica a si mesmo."
b. *Vis
k'ac-
k'ritik'eb-s?
tav-s
i-k'ritik'eb-s.
Quem.ACU homem-NOM critica-3sSUJ? REFL-ACU VPR-criticar-3sSUJ.

Lubowicz (1999) explica essas diferenças como estando relacionadas ao foco da construção reflexiva. A estratégia siebie - ou POSS+tav, para o georgiano - apresenta-se como a forma que permite a leitura de foco sobre objeto, o que a forma sie - ou -i-+tav - não permite. Fato também importante é o de que a forma sie, assim como o -i-georgiano, ocorre também em verbos anticausativos:

(20) Drzwie sie otworzyly.

Porta se abriu.

"A porta abriu."

Entretanto, é necessária uma explicação de por que o morfema -itambém não ocorre com tav nas reflexivas, ou o contrário: por que tav ocorre nas construções reflexivas? 
Dois desenvolvimentos dentro do Programa Minimalista são bastante relevantes ao se tratar de construções reflexivas: a Teoria de Checagem e a ausência do nível de representação Estrutura Profunda ou DS. Esses desenvolvimentos permitem que papel temático seja compreendido como um traço a ser satisfeito por checagem. Em Hornstein $(1999,2001)$, encontra-se uma interessante proposta com relação aos reflexivos, baseada justamente nessas propriedades sugeridas pelo Programa Minimalista: essas construções podem ser consideradas como o resultado da ocorrência de cópias do argumento interno do verbo à posição de argumento externo para satisfazer os papéis temáticos requeridos. Um desenvolvimento dessa proposta, visto em Alboiu et al. (2004), utiliza-se também das propriedades da Morfologia Distribuída para explicar as estratégias de reflexivização nas línguas românicas.

Um argumento interessante para esse tipo de abordagem é justamente o de que ele prevê o comportamento das reflexivas como tendo propriedades inacusativas e inergativas. Há uma grande discussão na literatura a respeito disso, e o que tem ocorrido é que alguns autores consideram reflexivas como um tipo de construção inacusativa (BOUCHARD, 1984; EMBICK, 1998, 2004; GRIMSHAW, 1990; MARANTZ, 1984, entre outros) ou como um tipo de verbo inergativo (CHIERCHIA, 1989; REINHART, 1997, 2000; REINHART; SILONI, 2000, etc.). Porém, uma abordagem como a proposta em Hornstein $(1999,2001)$ e Alboiu et al. (2004) acaba por explicar justamente a causa dessa discussão: reflexivos são o resultado do movimento de um argumento interno do verbo até a posição de sujeito (passando por argumento externo), o que os dá certas características de inacusativos, porém esse argumento também satisfaz o papel temático de argumento externo. Isso explica o fato de que verbos reflexivos passam tanto por alguns testes de inacusatividade como por alguns de inergatividade.

O mais interessante dessa teoria, no entanto, é justamente seu potencial para estabelecer o status do nome tav nas construções reflexivas. Isso fica claro se observamos a estratégia de reflexivização do inglês que é pronome+self (i.e. my-self, your-self, etc.) - semelhante ao georgiano. Para Hornstein (2001), a ocorrência de self é explicada devido ao congelamento que um DP sofre para movimento-A quando satisfaz Caso (cf. Chomsky, 2001, 2004). Esse congelamento ocorreria em um DP argumento interno que satisfizesse seu Caso, impedindo-o de mover para outras posições argumentais - justamente o que ocorre nas reflexivas segundo a análise em questão. Para tanto, Hornstein (2001) postula self como um morfema que checa o Caso e deixa o DP livre para o movimento. Como mostrarei adiante, a ausência de tav em verbos anticausativos, por si, constitui um argumento para que se estabeleça tav como um correspondente georgiano de self.

A proposta de Hornstein pode ser implementada ao georgiano se assumimos que o morfema -i- é a realização de uma cópia baixa do sujeito das reflexivas, da mesma forma que o pronome possessivo. Isso implica dizer que -i- não é um operador, e sim um argumento do verbo, que é para onde a argumentação realizada até agora nos encaminha. Uma questão importante agora é como que ocorre a realização dessa cópia baixa e por que sua forma é diferente da forma plena da cópia alta.

Alboiu et al. (2004) explicam isso por assumir, ao invés das condições B e C de ligação, a Condição Geral de Cadeias Argumentais (General Condition on A-Chains, doravante GCA) proposta em Reinhart \& Reuland (1993). Tal condição restringe a realização fonológica das cadeias por predizer que uma cadeia máxima $\left(\alpha_{1} \ldots \alpha_{n}\right)$ deve possuir apenas um elo $-\alpha_{1}-$ que seja ao mesmo tempo $+\mathrm{R}$ (totalmente independente referencialmente) e marcado com Caso. Uma sentença como $O \mathrm{João}_{i} s e_{i}$ viu é possível já que $s e$ não é +R: $s e$ é subespecificado em termos de traços- $\varphi$. Para dar conta do caso concreto do SE românico, os autores propõem - dentro do panorama teórico da Morfologia Distribuída - que há um empobrecimento nos traços- $\varphi$ do DP mais baixo, 
fazendo com que o expoente fonológico SE - especificado apenas para pessoa e número - seja inserido. Para o georgiano pode-se dizer o mesmo: -i- ou o pronome possessivo são expoentes fonológicos que se inserem em cópias defectivas do sujeito. $O$ esquema abaixo ilustra o processo, onde AE significa Argumento Externo e AI, Argumento Interno:

(21) a. Após checagem de traços:

\begin{tabular}{llll} 
Spec, TP & \multicolumn{1}{c}{$\mathrm{AE}$} & $\mathrm{AI}$ & \\
$\mathrm{Ivan}_{\mathrm{i}}$ & $\mathrm{Ivan}_{\mathrm{i}}$ & $\mathrm{Ivan}_{\mathrm{i}}$ & - Formas abstratas \\
$\mathrm{NOM}$ & & $\mathrm{ACU}$-tav & - tav checa caso acusativo \\
$\varphi$-completo & $\varphi$-completo & $\varphi$-completo & \\
Após Spell-Out (Caminho para PF) & \\
Spec, TP & $\mathrm{AE}$ & $\mathrm{AI}$ & \\
$\mathrm{Ivan}_{\mathrm{i}}$ & & $-\mathbf{i}_{-}$ & - Inserção de fonologia \\
NOM & tavs & & \\
$\varphi$-completo & $\varphi$-completo & $\varphi$-incompleto & - Empobrecimento
\end{tabular}

É importante que se note que, nesse panorama, todo DP marcado com Caso precisa ser realizado. Nesse sentido, o empobrecimento da cópia inferior é forçado por um requerimento desse tipo ${ }^{10}$. Nesse momento, porém, o que necessita ser explicado é a ocorrência de -i- nos verbos anticausativos. Nota-se que os verbos anticausativos são sintaticamente inacusativos, o que significa que há um movimento A do argumento interno para a posição de sujeito. Entretanto, tal movimento acontece justamente porque o argumento interno não pode checar seu Caso naquela posição. A falta de caso acusativo ${ }^{10}$ Essa é a proposta de Hornstein. Mas Alboiu et al. (2004) também apontam em nota para outros
requisitos que levem a cópia inferior a ter de ser realizada, como o de "Transitividade" proposto por
Bowers (2002). justifica a ausência do tav nessas construções, como se vê abaixo. No entanto, não se explica o porquê de -i- ocorrer, visto que se referiria a uma cópia sem Caso.

(22) a. Lud-i da-i-mal-a.

Cerveja-ABs PVB-VPR-sumir-3sSUJ.AOR.

"A cerveja sumiu."
b. ${ }^{*}$ Lud-i
tav-i
da-i-mal-a.
Cerveja-ABS REFL-ABS PVB-VPR-sumir-3sSUJ.AOR.

Embora -i- ocorra em anticausativos, seu alternante, o pronome possessivo, não pode ocorrer, conforme ilustrado abaixo:

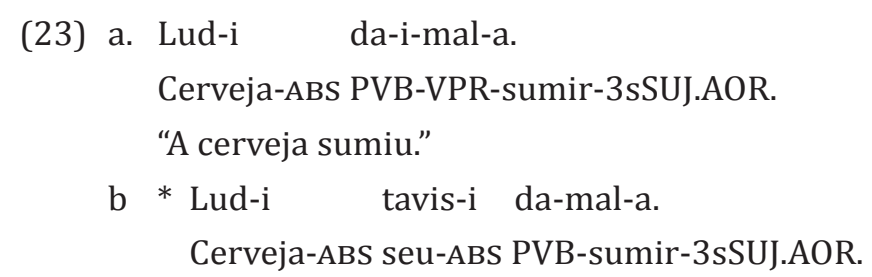

O que impede o pronome possessivo de ocorrer é justamente o fato de este ocupar uma posição argumental que requeira Caso checado. A vogal pré-radical -i- parece, por outro lado, ocorrer, por ser um morfema. Em termos de Morfologia Distribuída, pode-se dizer que há uma operação que adjunge uma cópia do argumento interno ao radical verbal e que, nessa posição, a cópia é invisível à GCA. Esse tipo de adjunção já havia sido proposto em Marantz (1988), com o nome de Morphological Merger. Atualmente diz-se que essa operação pode ocorrer durante a derivação sintática, para fins de checagem de traços. A postulação original da teoria encontra-se a seguir: 
(24) Merger Morfológico: em qualquer nível de análise da Sintaxe uma relação entre $\mathrm{X}$ e Y pode ser substituída pela afixação do núcleo $\mathrm{X}$ ao núcleo $\mathrm{Y}^{11}$.

Considerando que essa operação diz respeito a uma relação binária entre nós terminais X e Y, sua realização entre um verbo e um nome para efeitos de checagem de algum traço exclui, por exemplo, que essa checagem seja feita também por qualquer outro nome que esteja coordenado dentro do objeto verbal. Isso explica o porquê de -i- não poder ocorrer em coordenação nas construções reflexivas, por exemplo:

$$
\begin{aligned}
& \text { (25) * K'ac-i mariam-s da tav-s i-k'ritik'eb-s. } \\
& \text { Homem-Nom Maria-ACU e REFLX-ACU VPR-criticar-3sSUJ. } \\
& \text { "O homem critica a Maria e a si mesmo." }
\end{aligned}
$$

Alguém poderia, no entanto, dizer que essa operação extra resultaria em maiores custos para a análise. Entretanto, não se trata de uma operação extra. Se retomamos Lubowicz (1999), a explicação para a diferença de comportamento entre sie e siebie - que é a mesma entre -i-+tav e POSS+tav - está no foco. 0 primeiro caso apresenta foco sobre atividade e o segundo, foco sobre objeto. Supondo que a operação ocorra entre um nome que cheque seu traço de foco não-interpretável com um verbo de foco interpretável. Isso derivaria uma leitura de foco sobre atividade ${ }^{12}$. Nesse caso, LF interpretaria

${ }^{11} \mathrm{~A}$ postulação original do Merger Morfológico foi realizada previamente à proposta do Programa Minimalista e a consequente abordagem relacional das diferenças entre núcleos e projeções máximas e intermediárias de Bare Phrase Structure. Dadas essas diferenças do Programa Minimalista, considero, para os efeitos desta análise, que o Merger Morfológico pode realizar movimentos que não sejam necessariamente de núcleo.

${ }^{12}$ Um traço semântico não-interpretável, como o de foco que propomos aqui, pode soar como algo paradoxal. No entanto, pode-se compreendê-lo como a formalização da impossibilidade que o constituinte apresenta de ser interpretado de certa maneira. Uma formalização desse tipo é crucial para compreender julgamentos como em (26). foco apenas sobre o conjunto Verbo+nome-afixado, o que explicaria o seguinte dado apresentado anteriormente:

(26) a. Vis k'ac-i k'ritik'eb-s? k'ritik'eb-s tavis tav-s. Quem.ACU homem-NOM criticar-3sSUJ? criticar-3sSUJ seu.acu REFL-ACU. "Quem o homem critica? - critica a si mesmo."
b. *Vis
k'ac-i
k'ritik'eb-s? tav-s
i-k'ritik'eb-s.

Quem.Acu homem-NOM critica-3sSUJ? REFL-ACU VPR-criticar-3sSUJ.

Como se vê, a estratégia -i-+tav não pode responder a pergunta em (26b), já que, para isso, seria necessário um DP focalizado. Considerando que o argumento dos verbos anticausativos não apresenta foco sobre objeto - o sujeito realizado pode obter foco por outras estratégias - a adjunção do argumento interno ao verbo deve ocorrer necessariamente, ocasionando a inserção de -i-.

Na verdade, -i- é um elemento menos especificado do que o pronome possessivo. A ocorrência de pronomes possessivos requer foco sobre objeto, ou seja, requer que o DP objeto apresente um traço de foco interpretável. Isso é importante, uma vez que o Merger copia o argumento interno do verbo no caso de satisfação de foco sobre atividade. Se a cópia que restou do argumento interno - a cópia não adjunta ao verbo - checa o caso do verbo, ela deveria ser realizada como um pronome possessivo, juntamente com -i-, conforme ilustro abaixo:

$$
\begin{aligned}
& \sqrt{\mathrm{P}} \\
& \text { gp DPi }[u F O C]+\text { CASO } \\
& \text { qp /POSS } \\
& \mathrm{DPi}_{\mathrm{i}}[\mathrm{uFOC}] \quad \sqrt{\text { Verbo }}[\mathrm{Foc}] \\
& \text { /-i-/ }
\end{aligned}
$$


No entanto, pode-se dizer que os pronomes possessivos objetos sejam itens de vocabulário especificados para o traço de foco interpretável. A evidência para isso está nas construções transitivas com -i-, que eram um obstáculo para uma análise como a de Embick. Essas construções, em que -ise alterna com o pronome possessivo, apresentam as mesmas características de foco das reflexivas:

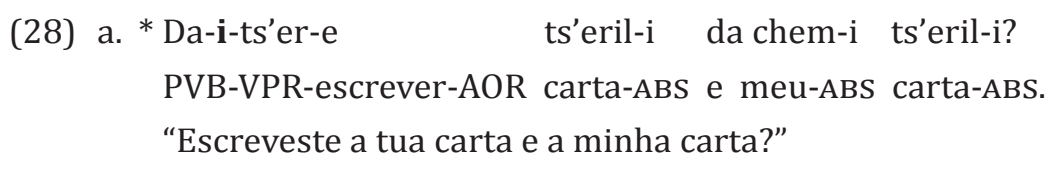

A operação de Merger para foco sobre atividade, na verdade, é algo bastante produtivo no georgiano e que está relacionado às próprias vogais pré-radicais que foram apresentadas no início deste texto. Até agora vimos o comportamento da vogal -i-. Entretanto, a vogal -a-, que indica locativos, e a vogal -u-, que indica argumentos beneficiários, também podem ser explicadas pelo mesmo motivo. Essas vogais, no entanto, alternam-se com preposições, conforme mostro abaixo:
(30)
Mama a-ts'er-s
ts'eril-s kaghald-s.

Pai.NOM VPR-escrever-3sSUJ carta-ACU papel-DAT.

"O Pai escreve a carta sobre o papel."
b. Mama ts'er-s
ts'eril-s kaghald-ze.

Pai.NOM escrever-3sSUJ carta-ACU papel-sobre.DAT

"O Pai escreve a carta sobre o papel."

(31) a. Is ga-u-k'et-a tavis deda-s.

Isso.ABS PVB-VPR-fazer-3sSUJ seu.DAT mãe-DAT.

"Ele fez isso para/pela sua mãe."
b. Is ga-ak'et-a
tavis-i ded-is-tvis
Isso.ABS PVB-fazer-3sSUJ seu-GEN mãe-GEN-para.
"Ele fez isso para/pela sua mãe."

Interessantemente, essas vogais terão as mesmas restrições vistas com -i-:

\section{(32) a. * Mama a-ts'er-s ts'eril-s kaghald-s da magida-ze.} Pai.NOM VPR-escrever-3sSUJ carta-ACU papel-DAT e mesa-DAT.sobre. "O Pai escreve a carta sobre o papel e sobre a mesa."

\section{b. Mama ts'er-s ts'eril-s kaghald-ze da magida-ze.}

Pai.Nom escrever-3sSUJ carta-ACU papel-sobre.DAT e mesa-DAT.sobre. "O Pai escreve a carta sobre o papel e sobre a mesa."

c. * Sad mama ts'er-s ts'eril-s? - a-ts'ers magida-s. Onde pai escreve-3sSUJ carta-AcU? - VPR-escrever-3sSUJ mesa-DAT. "Onde o pai escreve a carta? - Escreve sobre a mesa."

d. Sad mama ts'er-s ts'eril-s? ts-er-s magida-ze.

Onde pai escreve-3sSUJ carta-ACU? escrever-3sSUJ mesa-DAT.sobre. "Onde o pai escreve a carta? - Escreve sobre a mesa." 
(33) a. *Vistvis mas ak'eteb? - v-u-ket'eb deda-s.

Para-quem isso.Acu fazer? - 1sSUJ-VPR-fazer mãe-DAT.

"Para quem você está fazendo isso? - Estou fazendo para a minha mãe."

b. Vistvis mas ak'eteb? - v-aket'eb ded-is-tvis.

Para-quem isso.AcU fazer? -1sSUJ-fazer mãe-GEN-para

"Para quem você está fazendo isso? - Estou fazendo para a minha mãe."

Isso indica que realmente, para o georgiano, parece haver um processo morfológico relacionado a ausência de foco sobre objeto que copia esse objeto para uma posição de adjunção ao verbo. Nota-se que, assumindo que a inserção fonológica se dá tardiamente - seguindo o modelo da Morfologia Distribuída -, o conteúdo copiado para checar foco não necessariamente precisa ser apenas a preposição ou o pronome possessivo dos objetos. Se o objeto inteiro for copiado, ainda assim terão realização fonológica apenas os traços para os quais as vogais estão especificadas, ou seja, os traços de pronome possessivo ou de preposição. Os nomes não podem ser realizados uma vez que não estão em posição de checagem de Caso. 0 esquema abaixo ilustra isso:

(34)

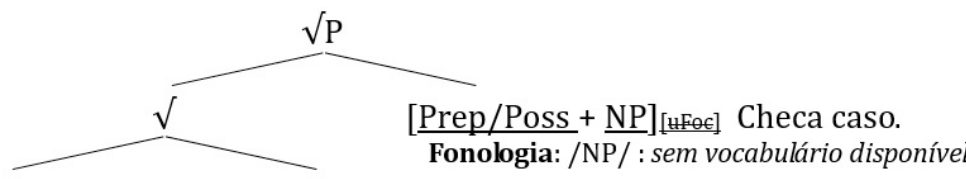

$[\underline{\text { Prep/Poss }}+\underline{\mathrm{NP}}]_{[\amalg F o c]}$ Verbo[Foc] para Prep/Poss, já que não apresenta Foco.

Fonologia: /Prep/Poss/ Sem

realização do NP, já que não

checa Caso nessa posição.

\section{Considerações Finais}

Em síntese, foi possível mostrar nesse texto que - ao contrário do que foi proposto em Nash (2002) - as vogais pré-radicais, e o -i- em especial, não são operadores de valência verbal. A alternância que o morfema -i- apresenta com pronomes possessivos e as diferenças na distribuição das estratégias de reflexivização -i-+tav e POSS+tav são evidências de que o morfema é a contraparte não-focalizada do pronome possessivo. A ocorrência da alternância entre as demais vogais pré-radicais e preposições motivadas pelos mesmos fenômenos de focalização constitui uma evidência independente para a análise proposta para a vogal -i-: o georgiano realiza uma operação de adjunção do objeto ao verbo sempre que esse objeto não obtiver foco. Nessa análise, a disponibilidade de vocabulário específico para traços de pessoa, locação ou benefício na posição de adjunção ao verbo garante a inserção das vogais pré-radicais. Prova disso são os verbos anticausativos, que, por não apresentarem objeto focalizado, têm seu argumento interno copiado à adjunção verbal, realizando-se, portanto, a vogal -i-.

\section{Referências}

ALBOIU, Gabriela; BARRIE, Michael; FRIGENI, Chiara. SE and the unaccusative-unergative paradox. In: COENE, Martin; CUYPER, Gretel; D’HULST, Yves (Org.). Antwerp Papers in Linguistics 107. Antwerp: Universiteit Antwerp, 2004. p. 109-139.

BOUCHARD, Denis. On the Content of Empty Categories. Dordrecht: Foris. 1984.

BOWERS, John. Transitivity. Linguistic Inquiry, Cambridge, Mass., v. 2, n. 33, p. 183-225, 2002. http://dx.doi.org/10.1162/002438902317406696

CHOMSKY, Noam. Derivation by Phase. In: KENSTOWICZ, Michael (Org.). Ken Hale: A Life in Language. Cambridge, Mass.: MIT Press. 2001. p. 1- 52.

CHOMSKY, Noam. Beyond Explanatory Adequacy. In: BELLETI, Adriana (Org.). Structures and Beyond. Oxford: Oxford University Press, 2004. p. 104-131.

EMBICK, David. Voice Systems and the Syntax/Morphology Interface. In: HARLEY, Heidi (Org.). The proceedings of the Penn/MIT workshop on Aspect, Argument Structure and Events. Cambridge, Mass: MIT Press, 1998.

GRIMSHAW, Jane. Argument Structure. Cambridge, Mass: MIT Press. 1990. 
HORNSTEIN, Norbert. Movement and Control. Linguistic Inquiry, Cambridge, Mass., v. 1, n. 30, p. 69-96, 1999. http://dx.doi.org/10.1162/002438999553968

HORNSTEIN, Norbert. Move! A Minimalist Theory of Construal. Oxford, UK/Cambridge, Mass.: Blackwell Publishers. 2001.

LAZZARINI-CYRINO, João. Paulo. O Sincretismo Passivo-Reflexivo em Georgiano: Discussões sobre Abordagens Formais. 2011. 127 f. Dissertação (Mestrado em Letras) Universidade Federal do Rio Grande do Sul, Porto Alegre, 2011.

LUBOWICZ, Anna. Two views of Polish reflexives. In: CARNIE, Andrew (Org.). WCCFL 18 Proceedings. Somerville, Mass.: Cascadilla Press, 1999. p. 337-350.

MARANTZ, Alec. On the Nature of Grammatical Relations. Cambridge, Mass.: MIT Press, 1984.

MARANTZ, Alec. Clitics, morphological merger, and the mapping to phonological structure. In: HAMMOND, Michael; NOONAN, Michael (Org.). Theoretical Morphology: Approaches in Modern Linguistics. San Diego: Academic Press, 1988. p. 253-270.

NASH, Lea. Entre la Flexion et le Verbe: syntaxe, morphologie, acquisition. Tese (Habilitação) - Universidade Paris VII, Paris, 2002.

REINHART, Tania. The theta system: syntactic realization of verbal concepts. Utrecht: OTS Working Paper, 2000.

REINHART, Tania; REULAND, Eric. Reflexivity. Linguistic Inquiry, Cambridge, Mass, v. 4, n. 24, p. 657-720, 1993. http://dx.doi.org/10.1.1.468.8796

REINHART, Tania; SILONI, Tal. The Lexicon-Syntax Parameter: Reflexivization and Other Arity Operations. Linguistic Inquiry, v. 3, n. 36, p. 389-436, 2005. http://dx.doi. org/10.1162/0024389054396881

Recebido em 04/04/2015.

Aceito em 12/06/2015. 